\title{
EVALUATION OF ABERRATIONS IN THE OPTICAL SYSTEM OF THE HUMAN EYE BASED ON THE SPATIAL SPECTRUM OF A DIAGNOSTIC IMAGE
}

\author{
N.Yu. Ilyasova ${ }^{1,2}$, D.A. Abulkhanov ${ }^{2}$, A.V. Kupriyanov ${ }^{1,2}$, A.V. Karsakov ${ }^{2}$ \\ ${ }^{1}$ Image Processing Systems Institute - Branch of the Federal Scientific Research Centre "Crys- \\ tallography and Photonics" of Russian Academy of Sciences, Samara, Russia \\ ${ }^{2}$ Samara National Research University, Samara, Russia
}

\begin{abstract}
This study deals with the analysis of wavefront aberrations that occur in the image owing to curvature variations in the human eye cornea. The analysis is based on the Liou-Brennan human eye model. The surface curvature and wavefront aberrations are described using Zernike polynomials. Our approach involves evaluating parameters of the distorting system's transfer function and measuring the expansion coefficients based on pattern recognition methods. Constructing a feature space of medical images enables the evaluation of aberrations in the eye optical system to be performed.
\end{abstract}

Keywords: aberrations in the human eye, Zernike polynomials, image spectral analysis.

Citation: Ilyasova NYu, Abulkhanov DA, Kupriyanov AV, Karsakov AV. Evaluation of aberrations in the optical system of the human eye based on the spatial spectrum of a diagnostic image. CEUR Workshop Proceedings, 2016; 1638: 39-48. DOI: 10.18287/1613-0073-2016-1638-39-48

\section{Introduction}

Our study aims to address a problem of the differential diagnosis of the human eye optical structures through analyzing higher-order aberrations of the focusing system. The human eye has been known to provide the major proportion of information about the world [1-4]. Impaired eye-vision deteriorates the quality of life. The immense experience science has gained in the treatment of eye diseases indicates that a key culprit is age-related changes in the eye optics, which can be evaluated based on wave aberrations. Higher-order (higher than astigmatism-related) aberrations cannot be corrected for and detected by standard optical techniques, while patients may complain of an impaired vision. At the same time, the eye may be affected by opticalsystem-unrelated diseases which can also have a potentially adverse effect on the vision. If higher-order aberrations and nonoptical abnormalities occur simultaneously 
it becomes impossible to make a differential diagnosis in practice in each individual case. Measurements of optical aberrations in the human eye are also of great importance in eye surgery. The possibility to detect optical aberrations during a routine check-up would enable clinical data to be adequately interpreted, while reducing the number of errors in choosing treatment tactics.

To address the above-described problems, a variety of optical models of the human eye have been proposed [5-7], which include dozens of parameters of refractive surfaces and eye tissues. Most often encountered in the literature is the eye model developed by Liou and Brennan in 1997 [5]. As a key innovation, this model takes into account the gradient refractive index of the crystalline lens. Currently, optical research has been under way aimed at improving the existing human eye models via introducing special aspherics on different surfaces and accounting for modern biometric measurements. One of key objectives is selecting eye model parameters based on the data of ophthalmological examination of an eye [7]. Based on medical images, the quality of an image formed in the eye can be evaluated, the disease diagnosed, and a treatment mode recommended.

\section{Image construction model}

In fig. 1 the conditional diagram of an eye explaining the course of rays in case of image generation is shown.

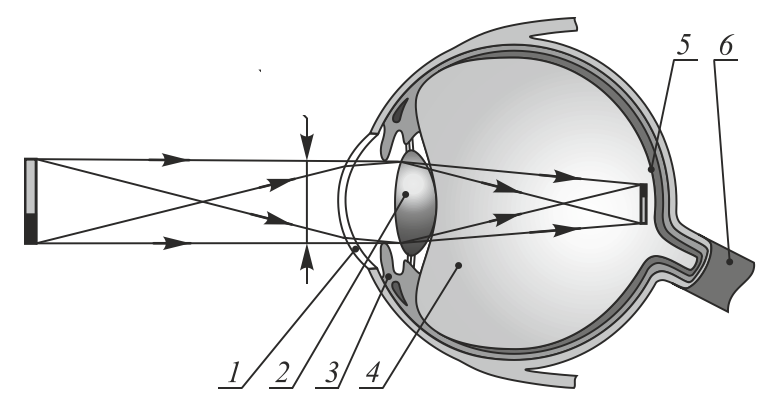

Fig. 1. Scheme eye image forming: 1 - cornea, 2 - crystalline lens, 3 - iris, 4 - vitreous body, 5 - retina, 6 - optic nerve

In Ref. [7] it was studied in Zemax in which way distortions of various refracting surfaces affected the quality of an imaging system of the human eye. The study was based on the Liou-Brennan human eye model with a gradient crystalline lens (Fig 2) [5]. 


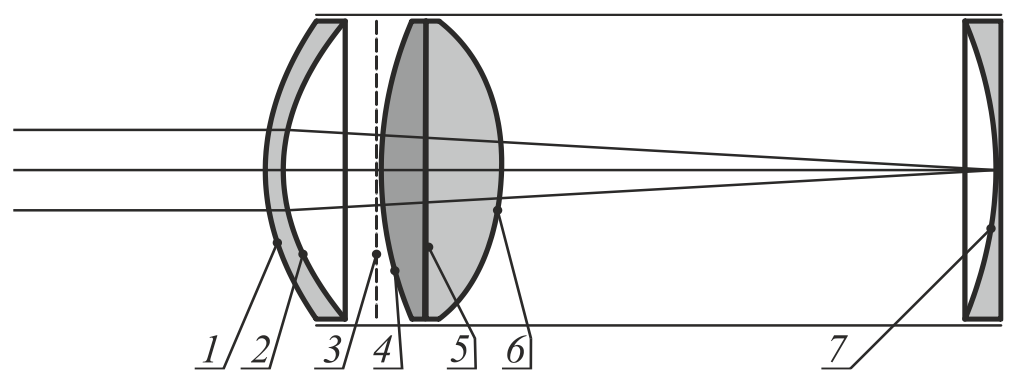

Fig. 2. A schematic layout of the refracting surfaces of a human eye according to the LiouBrennan model: 1- front corneal surface, 2- rear corneal surface, 3 - iris with a pupil, 4 - front crystalline lens surface, 5- interface between the crystalline lens' composite surfaces, 6- rear crystalline lens surface, and 7- retina.

The shape of the front cornea surface was simulated using a relationship composed of Zernike polynomials [8]:

$z(r, \varphi)=\frac{c r^{2}}{1+\sqrt{1-(1+k) c^{2} r^{2}}}+\sum_{i=1}^{N} A_{i} Z_{i}(\rho, \varphi)$,

where $c$ is the surface curvature (reciprocal of radius), $k$ is a conical constant ( $k=-\varepsilon^{2}$, where $\varepsilon$ is the eccentricity, with a hyperboloid of rotation being observed at $k<-1$, a paraboloid at $k=-1$, an ellipsoid at $-1<k<0$, or $k<0$, and a sphere at $k=0), N$ is the number of Zernike polynomials, $Z_{i}(\rho, \varphi)$ is the i-th Zernike polynomial in (2), $A_{i}$ is the weight of the $\mathrm{i}$-th Zernike polynomial, $\mathrm{r}$ is the radial coordinate, $\varphi$ is the angular coordinate, $\rho=r / R$ is the normalized ray coordinate, and $R$ is the normalization radius.

The Zernike polynomials are expressed as follows $[9,10]$. An even polynomial: $Z_{n}^{m}(\rho, \varphi)=R_{n}^{m}(\rho) \cos (m \varphi)$, an odd polynomial: $Z_{n}^{-m}(\rho, \varphi)=R_{n}^{m}(\rho) \sin (m \varphi)$, where

$R_{n}^{m}(\rho)=\sum_{k=0}^{\frac{n-m}{2}} \frac{(-1)^{k}(n-k) !}{k !\left(\frac{n+m}{2}-k\right) !\left(\frac{n-m}{2}-k\right) !} \rho^{n-2 k}$.

The wavefront aberrations can be expressed through Zernike polynomials [6]. Thus,

- Defocusing: $Z_{2}^{0}(\rho, \varphi)=\left(2 \rho^{2}-1\right)$,

- Third-order spherical aberration:

- $Z_{4}^{0}(\rho, \varphi)=\left(6 \rho^{4}-6 \rho^{2}+1\right)$,

- Distortion: $Z_{1}^{1}(\rho, \varphi)=\rho \cos \varphi$,

- Coma: $Z_{3}^{1}(\rho, \varphi)=\left(3 \rho^{3}-2 \rho\right) \cos \varphi$,

- Astigmatism $Z_{2}^{2}(\rho, \varphi)=\rho^{2} \cos 2 \varphi$. 
An output image of the diffractive aberration analyzer is composed of correlation peaks with their intensities being proportional to the contribution of the corresponding Zernike polynomial (and related aberration) to the wavefront under analysis. The angular dependence of the Zernike polynomials can be expressed via either cosinesine or exponential functions. In the latter case, wavefront aberrations can be detected irrespective of the angular rotation.

\section{$3 \quad$ Evaluating aberrations}

To obtain diagnostically significant information relating to aberration parameters of the human eye optical system, coefficients of the expansion in terms of Zernike polynomials need to be derived using digital image processing techniques.

Characterizing the root-mean-square deviation of the real wavefront from an ideal one, Zernike coefficients are used for obtaining quantitative characteristics of the optical image quality. The lower-order coefficients describe optical aberrations: defocusing (ametropia) and astigmatism. The higher-order coefficients define spherical aberration of slanted rays incident at an angle to the eye's optical axis. They stem from optical element asymmetry, leading to a shift of the cornea center relative to the crystalline lens and foveola center. Another contributing factor is a higher refractive power of the crystalline lens periphery when compared with its center for parallel incident rays. For known aberrations, it is possible to calculate the Strehl factor (defined as the ratio of the peak aberrated image intensity from a point source for the eye under analysis to the maximum intensity for an aberration-free eye) which correlates well with visual acuity under certain conditions. Based on the value of the Stehl factor, it becomes possible to prognosticate the vision acuity by simulating the imaging of any optotypes on the retina. In this work we aim to study in which way aberrations in the eye's focusing system affect the focal spot generated in the retina, as well as identifying the relationship between the eye condition diagnosed and the aberration coefficients measured using digital image processing techniques. We utilize machine learning methods using Zemax model images (Fig. 2), which simulate distortions associated with different corneal refractive surfaces in the Liou-Brennan eye model with a GRIN crystalline lens and are described by a particular Zernike polynomial. In the course of imaging simulation, the curvature of the front elliptical corneal surface is varied by introducing different Zernike polynomials with different weights into its components. This corresponds to the superposition of aberrations with weight coefficients that describe age-related changes and pathologies of the eye. Our idea is to evaluate the parameters of a transfer function of the distorting system and apply pattern recognition methods to measuring the coefficients of interest. To these ends, a feature-based description of distortion transforms needs to be formed using model data. Also, a classifier needs to be constructed using which it would be possible to identify aberrations, enabling the disease to be diagnosed.

The model data is in the form of gray-level 8-bit graphical images, derived via discretization of the output wavefront intensity in the eye model (Fig. 2). 


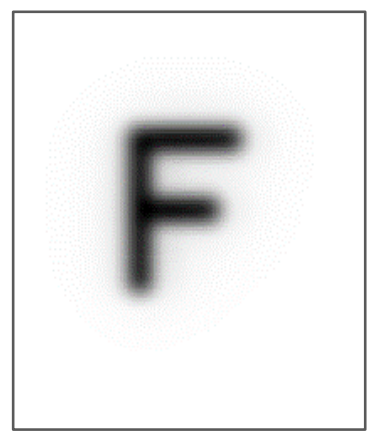

a)

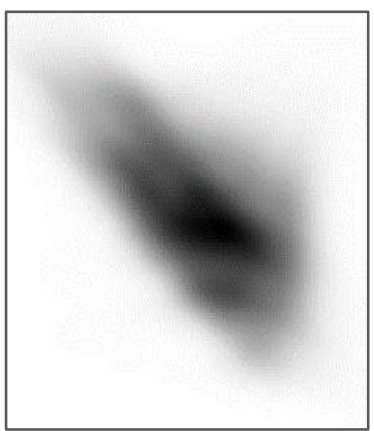

b)

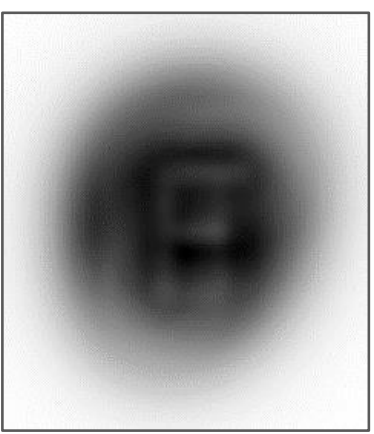

c)

Fig. 3. a) Original image and b,c) images distorted using different transforms.

To obtain a discrete spectrum of the initial and distorted images, let us preliminarily complement the 1024x1024 images with zeros, thus, obtaining 4096x4096 images (Fig. 4). As a characteristic of the distortion transform type, below we use a space of features derived by analyzing the transformation system's frequency response. In a matrix form, it can be written as $H_{i, j}=Y_{i, j} / X_{i, j}$ where $Y$ and $X$ are, respectively, matrices of a Fourier transform of the distorted and initial images, with the inversion and multiplication performed element-wise.

Figure 5 shows that owing to good discernibility of the image, the feature space can be in the form of an image scan of the amplitude of the frequency response's central part for the distortions under analysis viewed at different angles.

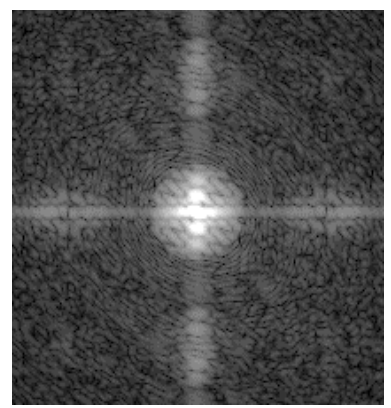

a)

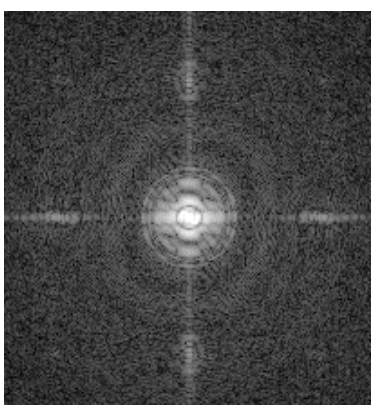

b)

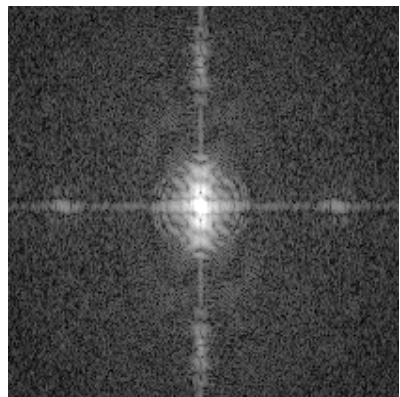

c)

Fig. 4. Amplitude of the central part of the Fourier transform of the images in Fig. 3.

Table 1 illustrates fragments of the frequency response amplitude images, which show good discernibility at different weights corresponding to Zernike polynomials. We propose using a method of principal components [11] enabling the feature space to be formed based on eigenvectors. 


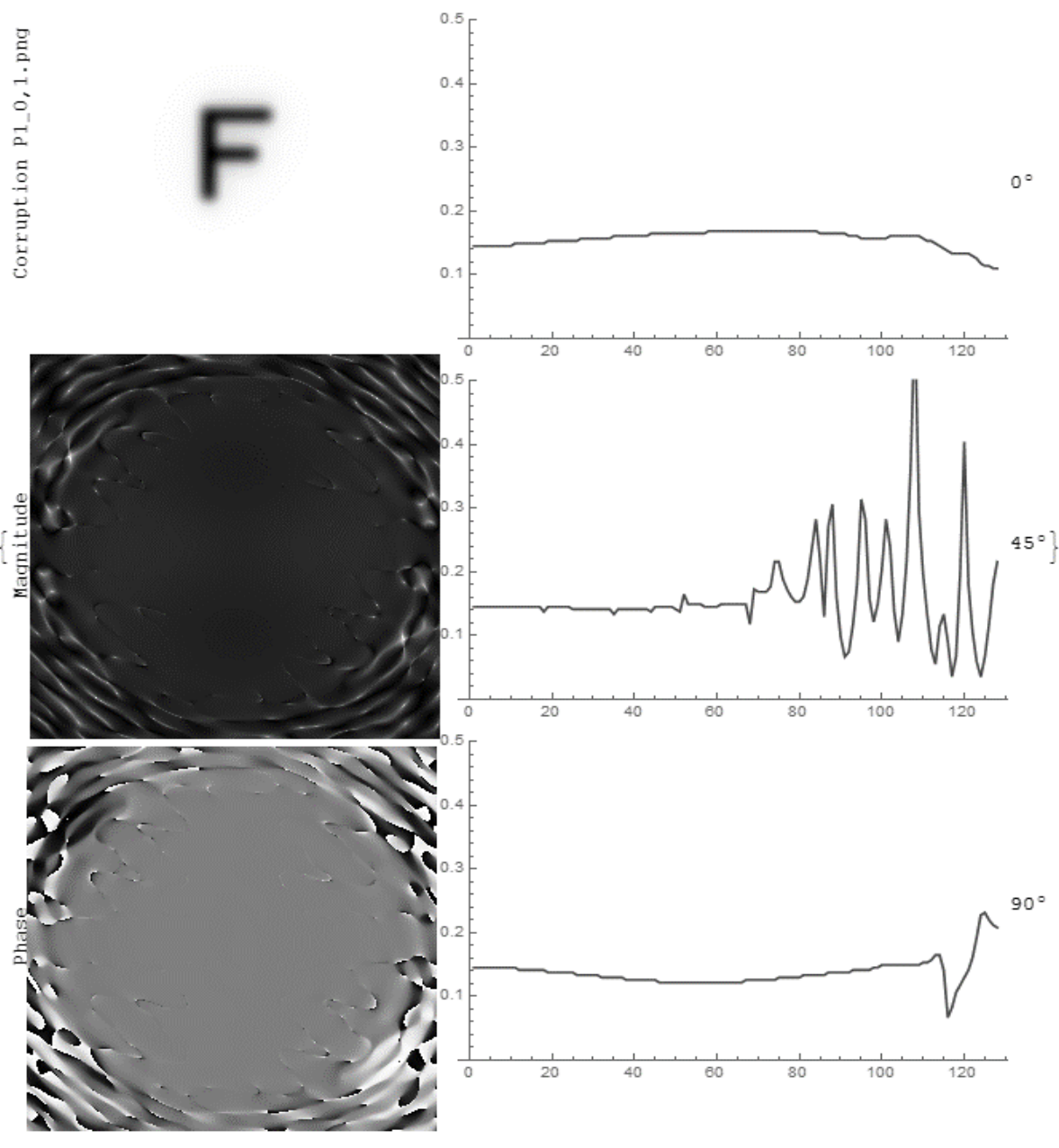

Fig. 5. Distorted images for different transformations, which are characterized by a lower contribution of the Zernike polynomial into the cornea curvature, the respective amplitude and phase of the frequency response's central part for a distortion transform and profiles of the frequency response amplitude taken at different angles $\left(Z_{2}^{-2}(\rho, \varphi)\right.$, weight $\left.A=0,1\right)$.

\section{$4 \quad$ Method of principal components}

For feature extracting we use Eigenfaces method [11] which produces dimension reduction by allowing the smaller set of basis images to represent the original training images. Eigenfaces are extracted out of the image data by means of principal component analysis (PCA) in the following manner: 

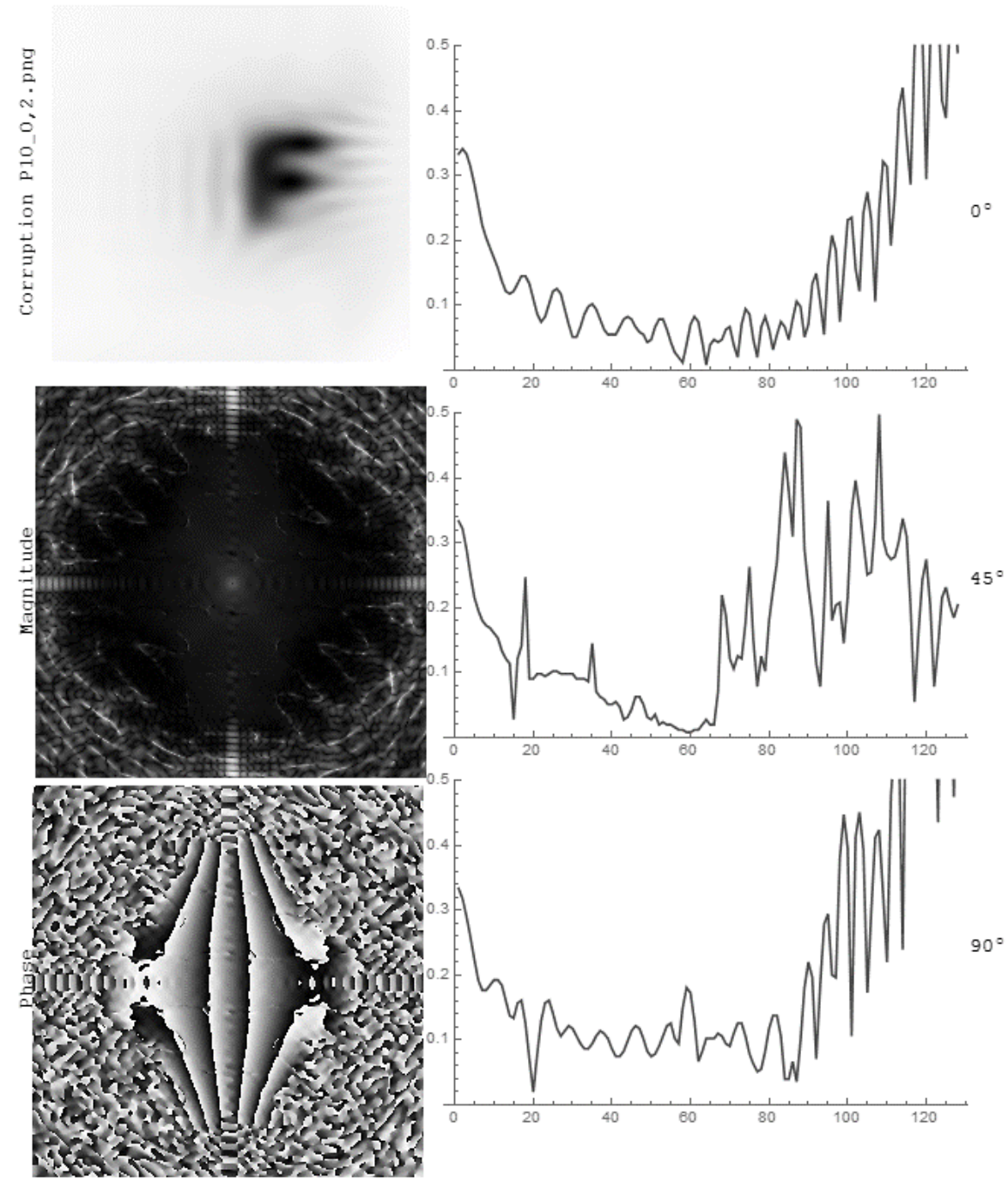

Fig. 6. Distorted images for different transformations, which are characterized by a higher contribution of the Zernike polynomial into the cornea curvature, the respective amplitude and phase of the frequency response's central part for a distortion transform and profiles of the frequency response amplitude taken at different angles $\left(Z_{2}^{-2}(\rho, \varphi)\right.$, weight $\left.A=0,2\right)$.

1. Given $N$ images with the size of $h \times w$, each image is transformed into a vector of size $m=h w$ and placed into the set $\left\{\mathrm{J}_{1}, \mathrm{~J}_{2}, \cdots, \mathrm{J}_{N}\right\}$.

2. Each vector differs from the average by the vector $\Psi_{i}=J_{i}-M$, where the average vector is defined by $M=\frac{1}{N} \sum_{i=1}^{N} J_{i}$. 
3. The covariance matrix $\mathbf{C} \in \mathbf{R}^{m \times m}$ is defined as $\mathbf{C}=\frac{1}{N} \sum_{i=1}^{N} \Psi_{i} \Psi_{i}^{\cdot}$.

4. Compute the eigenvalues $\lambda_{i}$ and eigenvectors $v_{i}$ of $\mathbf{C}$. We use SVD decomposition.

5. Order the eigenvectors descending by their eigenvalue. The eigenfaces (or eigenaberrations) are the eigenvectors corresponding to the largest eigenvalues.

Table 2 a) shows examples of the eigenvectors ('eigenfaces'), which can be termed as 'eigen-aberrations' and were derived based on the principal components corresponding to the maximum-value weight coefficients in the expansion. Table $2 \mathrm{~b}$ ) depicts expansions of the test images in terms of the eigen-aberrations in Table 2 a).

Table 1. Exemplary images of the frequency response amplitude of the fragments under analysis obtained for a) small weight and b) large weight.

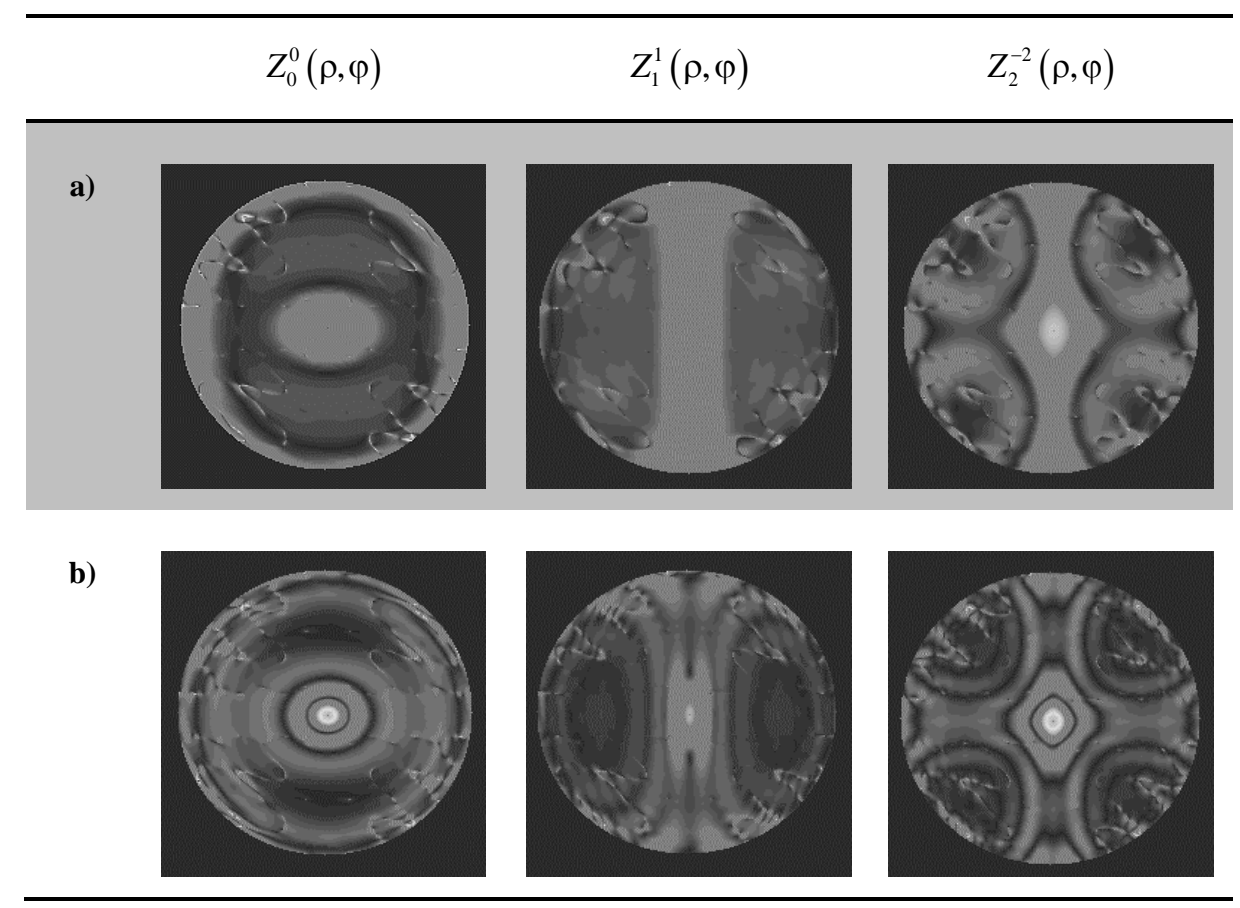

Summing up, we have shown that coefficients of an expansion in terms of eigenaberrations can be used as a feature space. A future study will be aimed at constructing and analyzing a classifier with use of the feature space proposed here. 
Table 2. (a) Examples of eigen-aberrations and (b) expansions in terms of the above eigenaberrations corresponding to maximum expansion coefficients.

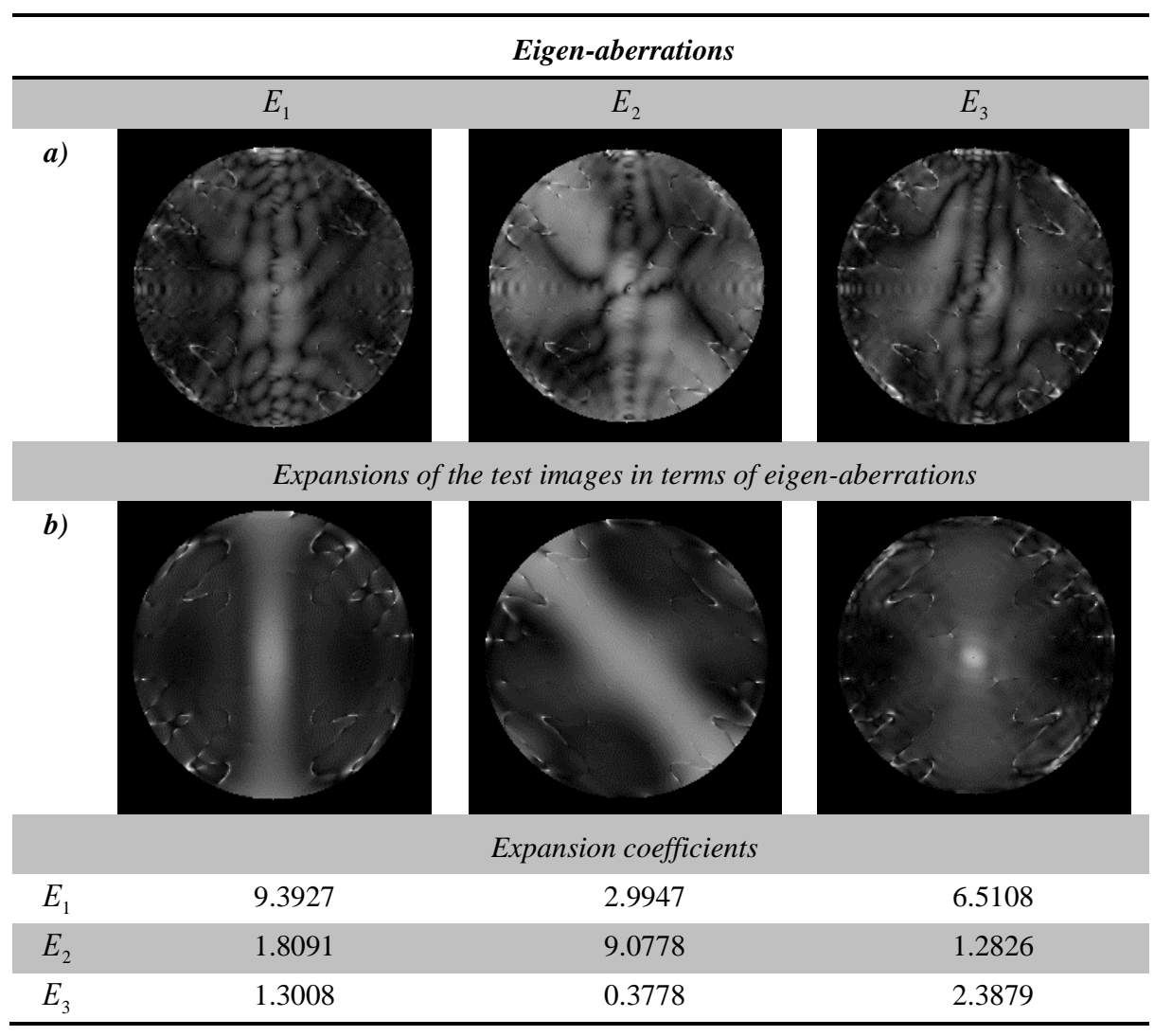

\section{Conclusion}

We have proposed a method for analyzing wavefront aberrations that occur in the image due to variations of the cornea surface curvature in the human eye. The analysis was based on a Liou-Brennan eye model. The surface curvature and wavefront aberrations were described in this work using Zernike polynomials. We have proposed that the transfer function parameters of a distorting system should be evaluated and pattern recognition methods used for measuring the expansion coefficients. A feature space of medical images has been constructed, enabling aberrations in the optical system of an eye to be evaluated.

\section{Acknowledgements}

The work has been performed with partial financial support from the Ministry of Education and Sciences of the Russian Federation within the framework of implementa- 
tion of the Program for Improving the SSAU Competitiveness among the World's Leading Research and Educational Centers for the Period of 2013-2020s; under the RFBR grants (the Russian Foundation for Basic Researches) 14-07-97040, 15-2903823, 15-29-07077, 16-57-48006; within the Basic Research Program No. 6 ONIT RAN of the Russian Academy of Sciences "Mathematical Methods and Information Technologies for the Analysis of Biomedical Images in Medical Diagnostics Applications" 2016.

\section{References}

1. Ilyasova NYu. Methods for digital analysis of human vascular system. Literature review. Computer Optics, 2013; 37(4): 517-541. [In Russian]

2. Ilyasova NYu. Computer Systems for Geometrical Analysis of Blood Vessels Diagnostic Images. Optical Memory and Neural Networks (Information Optics), 2014; 23(4): 278-286.

3. Ilyasova NYu, Kupriyanov AV, Paringer RA. Formation of features for improving the quality of medical diagnosis based on discriminant analysis method. Computer Optics, 2014; 38(4): 751-756. [In Russian]

4. Ilyasova NYu. Estimating the geometric features of a 3D vascular structureю Computer Optics, 2014; 38(3): 265-271. [In Russian]

5. Liou H-L, Brennan NA. Anatomically accurate, finite model eye for optical modeling. JOSA A, 1997; 14(8): 1881-91.

6. Bakholdin AV, Korshikova NF, Cherkasova DN. Computer simulation of an individual eye optical system. Izvestiya vysshikh uchebnykh zavedeniy. Priborostroenie, 2012; 55(4): 6873. [in Russian]

7. Degtyarev SA, Karsakov AV, Branchevskaya ES, Khonina SN, Kotlyar VV. Effects of variations in the refracting surfaces of the eye on the retinal image quality in the Liou-Brennan model. Computer Optics, 2015; 39(5): 702-708. [In Russian]. DOI: 10.18287/0134-24522015-39-5-702-708.

8. Oliveira CM, Ferreira A, Franco S. Wavefront analysis and Zernike polynomial decomposition for evaluation of corneal optical quality. J Cataract Refract Surg, 2012; 38(2):343-56.

9. Khonina SN, Kotlyar VV, Soifer VA, Wang Y, Zhao D. Decomposition of a coherent light field using a phase Zernike filter. Proceedings of SPIE, 1998; 3573: 550-553.

10. Khonina SN, Kotlyar VV, Kirsh DV. Zernike phase spatial filter for measuring the aberrations of the optical structures of the eye. Journal of Biomedical Photonics Engineering, 2015; 1(2): 146-153.

11. Turk M., Pentland A. Eigenfaces for Recognition. Journal of Cognitive Neuroscience, 1991; 3(1): 71-86. 